ORIGINAL ARTICLE

\title{
Effectiveness and Safety of Percutaneous Nephrolithotomy (PCNL) in Malrotated Kidneys
}

\author{
SAMI UR REHMAN ${ }^{1}$, LIAQAT ALI ${ }^{2}$, JEHANZEB ${ }^{3}$, MUHAMMAD ASIF $^{4}$, SYED ARIF $^{5}$, HANIF KHAN \\ ${ }_{1,5}$ Senior Registrar Urology department, Mian Gul Abdulhaq Jehanzab Kidney Center Sangota, Swat \\ ${ }^{2}$ Associate Prof Urology department, Institute of Kidney Diseases Hayatabad Medical Complex, Peshawar \\ ${ }^{3}$ Consultant Urologist, Institute of Kidney Diseases Hayatabad Medical Complex, Peshawar \\ ${ }^{4}$ Consultant Urologist, DHQ hospital, Mardan \\ ${ }^{6}$ Resident Urologist Institute of Kidney Diseases Hayatabad Medical Complex, Peshawar \\ Corresponding Author: Dr. Syed Arif, Email. drarif08@gmail.com, Cell No: +92 3339470932
}

\begin{abstract}
Background and Aim: The renal stones of any size could be effectively managed through an essential surgical technique known as Percutaneous Nephrolithotomy (PCNL). The large size renal stones with abnormal kidneys imposed additional challenges for PNCL in anomalous kidneys. The present study aimed to evaluate the effectiveness and safety of Percutaneous Nephrolithotomy in malrotated kidneys.

Place and Duration: Conducted at Urology department of Mian Gul Abdulhaq Jehanzeb Kidney Hospital Manglawar, Swat for duration of two years (from May 2019 to April 2021).

Materials and Methods. This single-centered retrospective study was conducted on 80 patients who underwent percutaneous nephrolithotomy with malrotation kidneys. The individuals with anomalous kidneys and complex calculi were enrolled in this study. These patients had kidneys anomalies such as horseshoe kidneys, crossed fused ectopia, malrotation kidneys, pelvic and complete stone clearance. Posterior or anterior approaches were followed for the procedure after preoperative evaluation in kidney anomalies. Retrograde catheterization was carried out under spinal and general anesthesia with the patients. For all the patients, stone size and clearance were measured.

Results: Of the total 80 kidneys anomalies patients, $55(68.7 \%)$ were male and $25(31.3 \%)$ were females. Overall mean age was $35.26 \pm 13.51$. The stone size varied from $1.3 \mathrm{~cm}$ to $7 \mathrm{~cm}$. Patients were categorized into two groups based on stone sizes such as group I $(1.3-2.5 \mathrm{~cm})$ had $42(52.5 \%)$ and group II $(25-7 \mathrm{~cm}) 38(47.5 \%)$ patients. The patients included renal pelvis $19(23.6 \%)$, the pelvic ureteric junction (PUJ) $8(10 \%)$, horseshoe kidneys $2(2.5 \%)$, crossed fused ectopia $9(11.3 \%)$, and malrotation kidneys $3(3.8 \%)$. The stone clearance was completed in $77(96.3 \%)$ patients. The statistically significant factor for complete clearance was the staghorn stone calculus.

Conclusion: Percutaneous nephrolithotomy is a safe and effective procedure for large renal stones management in patients of anomalous kidneys. Higher renal stones clearance can be achieved with minimum morbidity by suitable preoperative evaluation and technical experts.
\end{abstract}

Keywords: Malrotated kidney; Nephrostomy, Percutaneous; Nephrolithiasis; Abnormality

\section{INTRODUCTION}

Percutaneous nephrolithotomy is a promising treatment technique for renal stones with larger sizes above $2 \mathrm{~cm}$. However, urologists faced several challenges in complex anatomy or anomalies in performing PCNL operations. An effective and successful PCNL in terms of complete clearance and low complications can be established with easy and optimal access to the renal system $[1,2]$. However, because of the altered caliceal position, it is not always easy to achieve optimal access in malrotated kidneys, especially when fluoroscopy is used. Furthermore, due to the kidney's rotated anatomy, reaching the stone may be difficult. Horseshoe, ectopia without fusion and with fusion is the renal anomalies with kidney malrotation. Assessing the kidneys malrotation effect on other anomalies through PCNL could be challenging. The affection ratio in males is twice more compared to the female [3]. The PCNL success rate reported in various studies was above90\% [4, 5]. Upper ureteral and renal stones could be treated with effective and safe percutaneous nephrolithotomy [6]. The most common kidneys anomalies are malrotation, horseshoe with calculi presence, and fusion. The major challenges for horseshoe kidney treatment with percutaneous nephrolithotomy could be ectopia, lower pole fusion, and malrotation [7].

Anatomic anomalies, metabolic abnormalities, and urinary infections are the predisposing factors for PCNL. In developing countries, renal stones incidence are increasing in all age group globally [8]. Due to the low success rate and ureteric fragments of extracorporeal shock wave lithotripsy (ESWL) in renal calculi treatment and large size stones $(>1.5 \mathrm{~cm})$ percutaneous nephrolithotomy is the preferred technique for complex, large, and multiple stones. PCNL could be effective in renal anatomic variations and lower calyceal stones with a higher success rate of clearance and reduced morbidity [9]. PCNL safety and efficacy was investigated by various researchers among different age patients [10]. The present study aimed to evaluate the effectiveness and safety of Percutaneous Nephrolithotomy in malrotated kidneys.

\section{METHODS}

This single-centered retrospective study was conducted on 80 patients who underwent percutaneous nephrolithotomy with malrotation kidneys. The study was conducted at Urology department of Mian Gul Abdulhaq Jehanzeb Kidney Hospital Manglawar, Swat for duration of two years 
(from May 2019 to April 2021). The study was carried out after attaining approval from Institute Review Board. The individuals with anomalous kidneys and complex calculi were enrolled in this study. These patients had kidneys anomalies such as horseshoe kidneys, crossed fused ectopia, malrotation kidneys, pelvic and complete stone clearance. Posterior or anterior approaches were followed for the procedure after preoperative evaluation in kidney anomalies. Retrograde catheterization was carried out under spinal and general anesthesia with the patients. For all the patients, stone size and clearance were measured. A ureteral catheter $(6 \mathrm{~F})$ was inserted in the upper ureter after spinal and general anesthesia induction. The malrotated and horseshoe kidney compared to the normal kidney was lower and more vertical. Upper and middle pole posterior Calix was obtained in a single tract through percutaneous. A fiscal dilator (30F) was used with fascial dilators and subcostal dilation on am Platz sheath. For stone disintegration, pneumatic and ultrasonic lithotripsy with a nephroscope (24F) was introduced. Prior to the removal of stone fragments and percutaneous procedure, tubeless PNCL and nephrostomy $(20 \mathrm{~F})$ tubes were placed in different cases. After 48 hours of surgery, tubes were removed and hydrothorax or pneumothorax was ruled out through chest $x$-rays. Before the removal of the tube, a nephrostogram and plain KUB (kidney ureter and bladder) were done in all the patients.

The baseline and demographic characterizations such as stone location, size, and multiplicity were all recorded. Additionally, hemoglobin changes, stone-free rate, operative and hospitalized meantime, percutaneous frequency, analgesic requirement, complications, and residual stones subsequent treatment were all noted. Stone clearance, also known as stone-free rate (SFR), was defined as the absence of any residual fragments larger than $4 \mathrm{~mm}$ on postoperative KUB X-rays. A modified Clavin classification was used for postoperative complications assessment. Frequency and percentage were calculated for categorical variables using the chi-square test. Mean and standard deviation was calculated for continuous variables. A p-value of 0.05 was considered statistically significant.

\section{RESULTS}

Of the total 80 kidneys anomalies patients, 55 (68.7\%) were male and 25 (31.3\%) were females in Figure 1. Overall mean age was $35.26 \pm 13.51$. The stone size varied from $1.3 \mathrm{~cm}$ to $7 \mathrm{~cm}$. Patients were categorized into two groups based on stone sizes such as group I $(1.3-2.5 \mathrm{~cm})$ had $42(52.5 \%)$ and group II $(25-7 \mathrm{~cm}) 38(47.5 \%)$ patients as shown in Figure 2. The patients included renal pelvis 19 $(23.6 \%)$, the pelvic ureteric junction (PUJ) 8 (10\%), horseshoe kidneys 2 (2.5\%), crossed fused ectopia 9 $(11.3 \%)$, and malrotation kidneys 3 (3.8\%). The stone clearance was completed in $77(96.3 \%)$ patients. The statistically significant factor for complete clearance was the staghorn stone calculus.

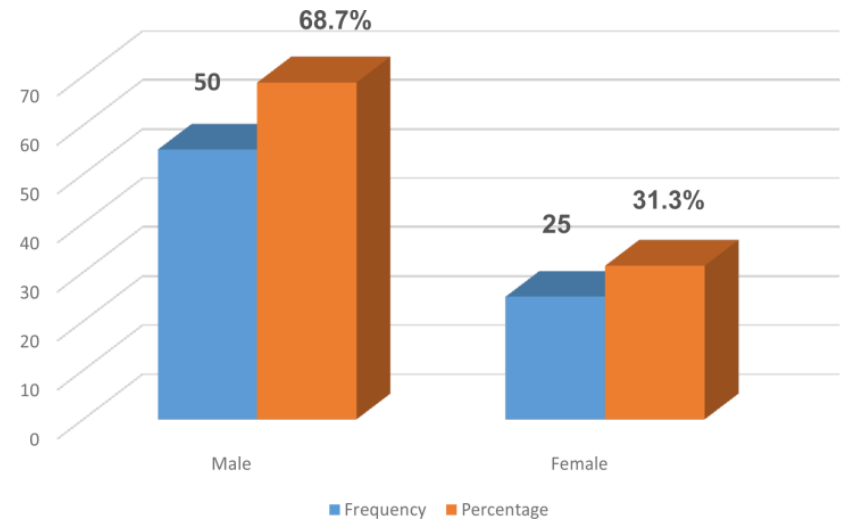

Figure 1. Gender distribution

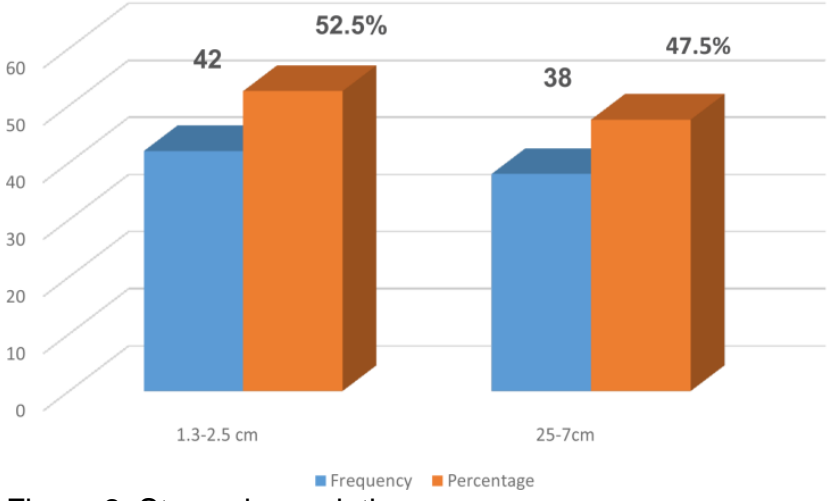

Figure 2. Stone size variations

Out of the 80 renal units, the prevalence of right and left kidneys stones were $43(53.8 \%)$ and 37 (46.2\%) respectively in Figure 3 . The stone location was renal pelvis $19(23.6 \%)$, pelvicalyceal $33(41.3 \%)$, calyceal 15 (18.6\%), and staghorn 13 (16.5\%) as shown in Table 1.

Table-1. Stone location distribution

\begin{tabular}{|l|l|l|}
\hline Stone location & Frequency (n) & Percentage (\%) \\
\hline Renal pelvis & 19 & 23.6 \\
\hline Pelvicalyceal & 33 & 41.3 \\
\hline Calyceal & 15 & 18.6 \\
\hline Staghorn & 13 & 16.5 \\
\hline Total & 80 & 100 \\
\hline
\end{tabular}

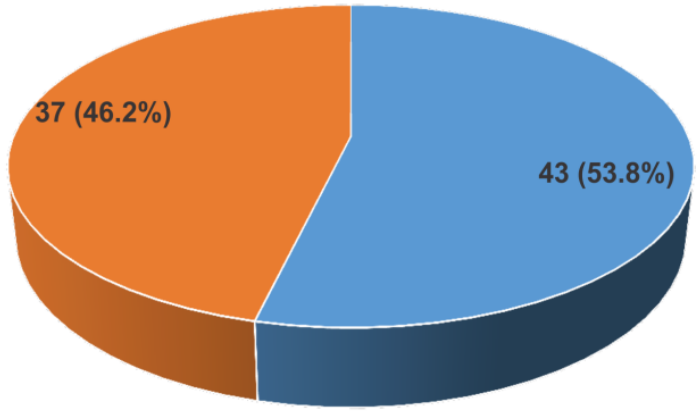

= Right Kidney = Left Kidney

Figure 3. Prevalence of right and left kidneys

Table 2 demonstrate the postoperative complications of PCNL were bladder clots 1 (1.3\%), fragment went into lower ureter $5(6.3 \%)$, leakage from puncture site $1(1.3 \%)$, stone went into injury outside kidney $3(3.8 \%)$ and no 
complications in 71 (89\%). The Guy's Stone Score (GSS) was calculated for all the patients and their prevalence as shown in Table 3.

Table 2. Postoperative complications

\begin{tabular}{|l|l|l|}
\hline Complications & Frequency (n) & Percentage (\%) \\
\hline Bladder clots & 1 & 1.3 \\
\hline $\begin{array}{l}\text { Fragment went into } \\
\text { lower ureter }\end{array}$ & 5 & 6.3 \\
\hline $\begin{array}{l}\text { Leakage from puncture } \\
\text { site }\end{array}$ & 1 & 1.3 \\
\hline $\begin{array}{l}\text { Stone went into injury } \\
\text { outside kidney }\end{array}$ & 3 & 3.8 \\
\hline No complications & 70 & 87.5 \\
\hline
\end{tabular}

Table-3 Frequency of Guy's Stone Score (GSS) among 80 patients

\begin{tabular}{|l|l|}
\hline Guy's Stone Score (GSS) & Frequency n (\%) \\
\hline 1 & $7(8.8)$ \\
\hline $1 \mathrm{a}$ & $9(11.3)$ \\
\hline $1 \mathrm{~b}$ & $27(33.8)$ \\
\hline $2 \mathrm{a}$ & $3(3.8)$ \\
\hline $2 \mathrm{~b}$ & $6(7.5)$ \\
\hline $2 \mathrm{c}$ & $3(3.8)$ \\
\hline $3 \mathrm{a}$ & $6(7.5)$ \\
\hline $3 \mathrm{~b}$ & $3(3.8)$ \\
\hline $3 \mathrm{c}$ & $10(12.2)$ \\
\hline $4 \mathrm{a}$ & $4(5)$ \\
\hline $4 \mathrm{~b}$ & $2(2.5)$ \\
\hline Total & $80(100)$ \\
\hline
\end{tabular}

\section{DISCUSSION}

The common types of renal abnormalities include malrotation anomalies and fusion practice of urology. The Shock Wave Lithotripsy (SWL) and flexible ureteroscopy are the most promising treatment modalities for renal stones management having less than $2 \mathrm{~cm}$. while PCNL is the most effective and safe technique for larger stone sizes more than $2 \mathrm{~cm}$. The prevalence of stone-free rate varied from $73-93 \%$ reported in various studies [11-13]. SWL is a failure or resistant cases modality while for larger stones, PCNL remains the standard reference. At autopsy, the ectopic kidneys incidence is approximately 1:900, with no gender preference. Fusion and abnormal placement result in a more anterior position of the renal pelvis, calyces that are more posterior than usual, and a combination of aberrant vasculature that can be extremely variable at times. The horseshoe kidney (1:400 in live births) with the abdomen lower fused pole, anterior pelvis, and vascular anomalies are the common anomalies of renal fusion. The higher stone formation prevalence and impaired drainage of the urinary are caused by higher insertion of the kidney ureter. The horseshoe cases account for $14 \%$ of stones [14].

Urolithiasis higher prevalence in anomalies kidneys caused by various parameters such as UPJ distortion/obstruction, pelvicalyceal position, concomitant infection, abnormal renal, aberrant renal vessels, complex calculi, metabolic abnormalities, and increasing stone burden. Other associated factors are skeletal difficulties and organs abnormalities in kidneys anomalies in many patients. Though small size renal stones $(<2 \mathrm{~cm})$ can be effectively managed by flexible ureteroscopy and SWL but
PCNL is a safe and effective treatment modality in large size stones $(>2 \mathrm{~cm})$. The major challenges of PCNL are calyceal access, puncture position, tract dilation, punctures number, and nephroscope mobility [15].

There are numerous studies in the literature on the management of stone disease in horseshoe kidneys. However, there is a scarcity of published data on PCNL studies that have included all types of anomalous kidneys. A study conducted on 16 complex calculi patients with anomalies kidneys found PCNL stone clearance 81\% [16]. Though, previously few published research has been carried out on horseshoe kidneys calculi management. A total of 200 renal malformations were reported with male predominance and renal stones varied from $234 \mathrm{~mm}-570$ $\mathrm{mm}$. The stone-free rate was reported at $77 \%$ [17]. In the majority of cases, infra-costal approach, superior calyceal punctures, and prone position was the main preference. The amplatz sheath size and tract dilation differ with the patient's stone burden, pelvicalyceal dilation degree, and age. PCNL anomalies kidneys in a total of 46 patients were administrated in operative time parameters [18]. Another study reported horseshoe kidney 85 , crossed fused ectopia 4 cases, pelvic kidneys 4 cases, and malrotation kidneys 7 cases [19].

The renal anomalies of PCNL calculus disease were reported high compared to the normal anatomical situation. Visceral anatomy of organ, pelvicalyceal configuration associated with anomaly types and kidney position, and vascular pathology varies with operative time. Multicalyceal stone location and stone burden were taken as key parameters varied with operative time [20]. The present study found insignificance effects of gender, age, calculi number and size, BMI, and renal anomalies types on stone-free rates. Other factors such as anomalous kidney morphology, large stone burden, stone location, pelvicalyceal the system, staghorn calculi were found statistically significant. The management of urolithiasis with larger stone size $(>2 \mathrm{~cm})$ in anomalous kidneys could be done safely and effectively through PCNL which reduced the morbidity. However, many challenges such as vascular anomalies, abnormal anatomy, restricted mobility, and visceral relationship interfere with the nephroscope. The proper assessment, cases selection, percutaneous renal surgery, and good training can manage PCNL anatomically abnormal kidneys.

\section{CONCLUSION}

Percutaneous nephrolithotomy is a safe and effective procedure for large renal stones management in patients of anomalous kidneys. Higher renal stones clearance can be achieved with minimum morbidity by suitable preoperative evaluation and technical experts.

\section{REFERENCES}

1. Goyal NK, Goel AS, Singh V, Singh BP, Sinha R. A critical appraisal of complications of percutaneous nephrolithotomy in paediatric patients using adult instruments. BJU Int. 2014;113:801-10.

2. Dongol UMS, Limbu Y. Safety and efficiency of percutaneous nephrolithotomy in children. J Nepal Health Res Counc. 2017;15(36):130-4.

3. Alomar MA, Alghamdi OS, Alghafees MA, Alhamidi RA, Abduldaem AM, Aljohani MM. Clinical Application of a Two- 
Step Percutaneous Nephrolithotomy in a Patient With Severe Kyphoscoliosis and a Malrotated Kidney. Cureus. 2021 Aug 20;13(8).

4. Omorogbe A, Rosen DC, Chandhoke R, Bamberger J, Gupta M. Ultrasound-Guided Access in a Malrotated Kidney: Optimally Safe and Effective. Journal of Endourology Case Reports. 2020 Sep 1;6(3):166-9.

5. Prakash G, Sinha RJ, Jhanwar A, Bansal A, Singh V. Outcome of percutaneous nephrolithotomy in anomalous kidney: Is it different? Urol Ann 2017;9:23-26.

6. Sabler IM, Katafigiotis I, Gofrit ON, Duvdevani M. Present indications and techniques of percutaneous nephrolithotomy: What the future holds? Asian J Urol 2018;5:287-294.

7. Mosavi-Bahar SH, Amirzargar MA, Rahnavardi M, Moghaddam SM, Babbolhavaeji $\mathrm{H}$, Amirhasani $\mathrm{S}$. Percutaneous nephrolithotomy in patients with kidney malformations. J Endourol 2007;21:520-524

8. Wang $\mathrm{K}$, Zhang $\mathrm{P}, \mathrm{Xu} \mathrm{X}$, Fan M. Ultrasonographic versus fluoroscopic access for percutaneous nephrolithotomy: a meta-analysis. Urol Int. 2015;95(1):15-25.

9. $\quad \mathrm{Ng}$ FC, Yam WL, Lim TYB, Teo JK, Ng KK, Lim SK. Ultrasound-guided percutaneous nephrolithotomy: advantages and limitations. Investig Clin Urol. 2017 Sep;58(5):346-52.

10. Thomas K, Smith NC, Hegarty N, Glass JM. The Guy's stone score: grading the complexity of percutaneous nephrolithotomy procedures. Urology. 2011 Aug;78(2):27781.

11. Tanidir Y, Sahan A, Asutay MK, Sener TE, Talibzade F, Garayev A, et al. Differentiation of ureteral stones and phleboliths using Hounsfield units on computerized tomography: a new method without observer bias. Urolithiasis. 2017 Jun;45(3):323-8.

12. Simon GI, Craswell A, Thom O, Fung YL. Outcomes of restrictive versus liberal transfusion strategies in older adults from nine randomised controlled trials: a systematic review and meta-analysis. Lancet Haematol. 2017 Oct;4(10):e46574.

13. Yang YH, Wen YC, Chen KC, Chen C. Ultrasound-guided versus fluoroscopy-guided percutaneous nephrolithotomy: a systematic review and meta-analysis. World J Urol. 2019 May;37(5):777-88.

14. Soares RMO, Zhu A, Talati VM, Nadler RB. Upper pole access for prone percutaneous nephrolithotomy: advantage or risk? Urology. 2019 Dec;134:66-71.

15. Singh R, Kankalia SP, Sabale V, Satav V, Mane D, Mulay A et al. Comparative evaluation of upper versus lower calyceal approach in percutaneous nephrolithotomy for managing complex renal calculi. Urol Ann. 2015 Jan-Mar;7(1):31-5.

16. Andonian S, Scoffone CM, Louie MK, Gross AJ, Grabe M, Daels FP, et al. Does imaging modality used for percutaneous renal access make a difference? A matched case analysis. J Endourol. 2013 Jan;27(1):24-8.

17. Tefekli A, Esen T, Olbert PJ, Tolley D, Nadler RB, Sun YH, et al. Isolated upper pole access in percutaneous nephrolithotomy: a large-scale analysis from the CROES percutaneous nephrolithotomy global study. J Urol. 2013 Feb;189(2):568-73.

18. Patel AP, Bui D, Pattaras J, Ogan K. Upper pole urologistobtained percutaneous renal access for PCNL is safe and efficacious. Can J Urol. 2017 Apr;24(2):8754-8.

19. Sourial MW, Francois N, Box GN, Knudsen BE. Supracostal access tubeless percutaneous nephrolithotomy: minimizing complications. World J Urol. 2019 Jul;37(7):1429-33.

20. Li J, Xiao B, Hu W, Yang B, Chen L, Hu H, et al. Complication and safety of ultrasound guided percutaneous nephrolithotomy in 8,025 cases in China. Chin Med J. 2014;127(24):4184-9. 